\title{
Mental disorders among college students in the World Health Organization World Mental Health Surveys
}

\author{
R. P. Auerbach ${ }^{1,2}$, J. Alonso ${ }^{3,4,5}$, W. G. Axinn ${ }^{6}$, P. Cuijpers ${ }^{7,8}$, D. D. Ebert ${ }^{9}$, J. G. Green ${ }^{10}$, I. Hwang ${ }^{11}$, \\ R. C. Kessler ${ }^{11 *}$, H. Liu ${ }^{12}$, P. Mortier ${ }^{13}$, M. K. Nock ${ }^{14}$, S. Pinder-Amaker ${ }^{1,2}$, N. A. Sampson ${ }^{11}$, \\ S. Aguilar-Gaxiola ${ }^{15}$, A. Al-Hamzawi ${ }^{16}$, L. H. Andrade ${ }^{17}$, C. Benjet ${ }^{18}$, J. M. Caldas-de-Almeida ${ }^{19}$, \\ K. Demyttenaere ${ }^{20}$, S. Florescu ${ }^{21}$, G. de Girolamo ${ }^{22}$, O. Gureje ${ }^{23}$, J. M. Haro ${ }^{24}$, E. G. Karam ${ }^{25,26,27}$, \\ A. Kiejna ${ }^{28}$, V. Kovess-Masfety ${ }^{29}$, S. Lee $^{30}$, J. J. McGrath ${ }^{31,32}$, S. O'Neill ${ }^{33}$, B.-E. Pennell ${ }^{34}$, K. Scott ${ }^{35}$, \\ M. ten Have ${ }^{36,37}$, Y. Torres ${ }^{38}$, A. M. Zaslavsky ${ }^{11}$, Z. Zarkov ${ }^{39}$ and R. Bruffaerts ${ }^{40}$
}

\begin{abstract}
${ }^{1}$ Department of Psychiatry, Harvard Medical School, Boston, MA, USA ${ }^{2}$ Center for Depression, Anxiety and Stress Research, McLean Hospital, Belmont, MA, USA; ${ }^{3}$ Institut Hospital del Mar d'Investigacions Mèdiques (IMIM), Barcelona, Spain; ${ }^{4}$ CIBERESP-CIBER en Epidemiología y Salud Pública, Madrid, Spain; ${ }^{5}$ Department of Experimental and Health Sciences, University Pompeu Fabra, Barcelona, Spain; ${ }^{6}$ Department of Sociology, Population Studies Center, Survey Research Center, Institute for Social Research, University of Michigan, Ann Arbor, MI, USA; ${ }^{7}$ Department of Clinical, Neuro, and Developmental Psychology, Vrije Universiteit Amsterdam, Amsterdam, The Netherlands; ${ }^{8}$ EMGO Institute for Health and Care Research, Amsterdam, The Netherlands; ${ }^{9}$ Department of Psychology, Clinical Psychology and Psychotherapy, Friedrich-Alexander University Nuremberg-Erlangen, Erlangen, Germany; ${ }^{10}$ School of Education, Boston University, Boston, MA, USA; ${ }^{11}$ Department of Health Care Policy, Harvard Medical School, Boston, MA, USA; ${ }^{12}$ Department of Epidemiology, Harvard T.H. Chan School of Public Health, Boston, MA, USA; ${ }^{13}$ Research Group Psychiatry, Department of Neurosciences, KU Leuven University, Leuven, Belgium; ${ }^{14}$ Department of Psychology, Harvard University, Cambridge, MA, USA; ${ }^{15}$ University of California Davis Center for Reducing Health Disparities, School of Medicine, Sacramento, CA, USA; ${ }^{16}$ College of Medicine, Al-Qadisiya University, Diwania Governorate, Iraq; ${ }^{17}$ Section of Psychiatric Epidemiology - LIM 23, Institute of Psychiatry, University of São Paulo Medical School, São Paulo, Brazil; ${ }^{18}$ Department of Epidemiologic and Psychosocial Research, National Institute of Psychiatry Ramón de la Fuente Muñiz, Mexico City, Mexico; ${ }^{19}$ Chronic Diseases Research Center (CEDOC) and Department of Mental Health, Faculdade de Ciências Médicas, Universidade Nova de Lisboa, Lisbon, Portugal; ${ }^{20}$ Department of Psychiatry, University Hospital Gasthuisberg, Katholieke Universiteit Leuven, Leuven, Belgium; ${ }^{21}$ National School of Public Health, Management and Professional Development, Bucharest, Romania; ${ }^{22}$ IRCCS St John of God Clinical Research Centre, Brescia, Italy; ${ }^{23}$ Department of Psychiatry, University College Hospital, Ibadan, Nigeria; ${ }^{24}$ Parc Sanitari Sant Joan de Déu, CIBERSAM, Universitat de Barcelona, Barcelona, Spain; ${ }^{25}$ Department of Psychiatry and Clinical Psychology, Faculty of Medicine, Balamand University, Beirut, Lebanon; ${ }^{26}$ Department of Psychiatry and Clinical Psychology, St George Hospital University Medical Center, Beirut, Lebanon; ${ }^{27}$ Institute for Development Research Advocacy and Applied Care (IDRAAC), Beirut, Lebanon; ${ }^{28}$ Department of Psychiatry, Wroclaw Medical University, Wroclaw, Poland; ${ }^{29}$ Ecole des Hautes Etudes en Santé Publique (EHESP), EA 4057 Paris Descartes University, Paris, France; ${ }^{30}$ Department of Psychiatry, Chinese University of Hong Kong, Tai Po, Hong Kong; ${ }^{31}$ Queensland Centre for Mental Health Research, The Park Centre for Mental Health, Wacol, Queensland, Australia; ${ }^{32}$ Queensland Brain Institute, The University of Queensland, St. Lucia, Queensland, Australia; ${ }^{33}$ School of Psychology, University of Ulster, Londonderry, UK; ${ }^{34}$ Survey Research Center, Institute for Social Research, University of Michigan, Ann Arbor, MI, USA; ${ }^{35}$ Department of Psychological Medicine, University of Otago, Dunedin, Otago, New Zealand; ${ }^{36}$ Trimbos-Instituut, Netherlands Institute of Mental Health and Addiction, Utrecht, the Netherlands; ${ }^{37}$ Department of Epidemiology, Netherlands Institute of Mental Health and Addiction, Utrecht, the Netherlands; ${ }^{38}$ Center for Excellence on Research in Mental Health, CES University, Medellin, Colombia; ${ }^{39}$ Department Mental Health, National Center of Public Health and Analyses, Sofia, Bulgaria; ${ }^{40}$ Universitair Psychiatrisch Centrum - Katholieke Universiteit Leuven (UPC-KUL), Campus Gasthuisberg, Leuven, Belgium
\end{abstract}

Background. Although mental disorders are significant predictors of educational attainment throughout the entire educational career, most research on mental disorders among students has focused on the primary and secondary school years.

Method. The World Health Organization World Mental Health Surveys were used to examine the associations of mental disorders with college entry and attrition by comparing college students $(n=1572)$ and non-students in the same age range (18-22 years; $n=4178)$, including non-students who recently left college without graduating $(n=702)$ based on surveys in 21 countries (four low/lower-middle income, five upper-middle-income, one lower-middle or upper-middle at the times of two different surveys, and 11 high income). Lifetime and 12-month prevalence and age-of-onset of DSM-IV anxiety, mood, behavioral and substance disorders were assessed with the Composite International Diagnostic Interview (CIDI).

\footnotetext{
* Address for correspondence: R. C. Kessler, Department of Health Care Policy, Harvard Medical School, 180 Longwood Avenue, Boston, MA 02115, USA.

(Email: kessler@hcp.med.harvard.edu)
} 
Results. One-fifth (20.3\%) of college students had 12-month DSM-IV/CIDI disorders; $83.1 \%$ of these cases had pre-matriculation onsets. Disorders with pre-matriculation onsets were more important than those with post-matriculation onsets in predicting subsequent college attrition, with substance disorders and, among women, major depression the most important such disorders. Only $16.4 \%$ of students with 12 -month disorders received any 12-month healthcare treatment for their mental disorders.

Conclusions. Mental disorders are common among college students, have onsets that mostly occur prior to college entry, in the case of pre-matriculation disorders are associated with college attrition, and are typically untreated. Detection and effective treatment of these disorders early in the college career might reduce attrition and improve educational and psychosocial functioning.

Received 11 April 2016; Revised 9 June 2016; Accepted 20 June 2016; First published online 3 August 2016

Key words: College attrition, college dropout, education, epidemiology, mental illness.

\section{Introduction}

Although prevalence (Costello et al. 2005; Merikangas et al. 2009) and treatment (Fazel et al. 2014a,b) of mental disorders among elementary and secondary school students has been the subject of considerable attention, less is known about mental disorder prevalence or treatment among college students other than in the USA (Eisenberg et al. 2007; Blanco et al. 2008; Cho et al. 2015; Kendler et al. 2015; Mojtabai et al. 2015). We know somewhat more about the associations of early-onset mental disorders with significant reductions in subsequent educational attainment (Kessler et al. 1995; Fergusson \& Horwood, 1998; Johnson et al. 1999; Miech et al. 1999; Woodward \& Fergusson, 2001; Fergusson \& Woodward, 2002; Fletcher, 2008; Lee et al. 2009; Mojtabai et al. 2015), but this work is limited by either being based on small restricted samples or by being subject to long-term recall bias. Given the importance of an educated workforce for the human capital potential of a country, it would be valuable to know more about five questions. First, what is the prevalence of mental disorders among college students? Second, what proportion of those disorders had onsets prior to college entry? Third, to what extent are disorders with prematriculation onsets associated with college entry? Fourth, what is the relative importance of disorders with pre-matriculation and post-matriculation onsets in predicting college attrition? Fifth, what proportion of college students with mental disorders receives treatment? We address these five questions using data from community epidemiological surveys carried out in 21 different countries in the World Health Organization (WHO) World Mental Health (WMH) Survey Initiative.

\section{Method}

\section{Samples}

The WMH surveys are a cross-national series of community epidemiological surveys using consistent sampling designs, field procedures and instruments to facilitate pooled cross-national comparative analyses (Kessler \& Üstun, 2011). The focus is on prevalence and correlates of common mental disorders. The data reported here come from the $23 \mathrm{WMH}$ surveys carried out in 21 countries that assessed college student status and had a sufficiently large sample to: (i) estimate prevalence among college students aged 18-22 years; and (ii) estimate and compare prevalence in disaggregated subsamples of non-students in the same age range as students, distinguishing college attriters from respondents who never entered college. The 1822 years age range was chosen because the vast majority of college students were in that age range across countries. We excluded college graduates because it was rare to find them in the 18-22 years age range.

The surveys were carried out in 21 countries: five classified by The World Bank (2012) as low- or lower-middle-income countries [national surveys in Colombia, Iraq and Peru, a regional survey in Nigeria, and regional surveys in Beijing-Shanghai and Shenzhen in the People's Republic of China (PRC)], six classified as upper-middle-income countries (national surveys in Bulgaria, Lebanon, Mexico and Romania and regional surveys in São Paulo, Brazil and Medellín, Colombia) and 11 classified as high-income countries (national surveys in Australia, Belgium, France, Italy, the Netherlands, New Zealand, Northern Ireland, Poland, Portugal, Spain and the USA). The national survey in Colombia was classified as lowermiddle income but the regional survey in Medellín, Colombia upper-middle income because the World Bank classification of Colombia's income level changed between the times of the two surveys.

Each WMH survey was based on a probability sample of household residents using a multi-stage clustered area probability sample design. Response rates ranged between 50.4\% (Poland) and 97.2\% (Colombia) with a weighted mean of $71.4 \%$ across surveys. A detailed description of sampling procedures is presented elsewhere (Heeringa et al. 2008). We focus here on all respondents in the age range of 18-22 
years who were either college students $(n=1572)$, college attriters $(n=702)$, secondary school graduates who never went to college $(n=1571)$ or people who never completed secondary school $(n=1905)$. We exclude the small number of 18- to 22-year-olds in the surveys who were college graduates $(n=183)$ because this was an uncommon outcome. In order to increase precision of prevalence comparisons, the joint agesex distributions of students and non-students within each country were standardized to equal the pooled student distribution across all countries combined. All results reported here combine men and women. However, all analyses were also carried out in separate samples of men and women (see online Supplementary material) and important differences in results are noted throughout the text.

\section{Field procedures}

Interviews were administered face-to-face in respondent homes (or, in the case of students living in campus group housing, in their student residences) after obtaining informed consent using procedures approved by local institutional review boards. All procedures used complied with the ethical standards of the relevant national and institutional committees on human experimentation and with the Helsinki Declaration of 1975 as revised in 2008. The interview schedule was developed in English and translated into other languages using a standardized WHO protocol (Harkness et al. 2008). Interviews were administered in two parts. Part I, administered to all respondents, assessed core Diagnostic and Statistical Manual of Mental Disorders, 4th edition (DSM-IV) mental disorders. Part II assessed additional disorders and correlates. Part II was administered to $100 \%$ of part I respondents who met lifetime criteria for any part I disorder and a probability subsample of other part I respondents. Part II respondents were weighted to adjust for differential probabilities of selection into part II, making estimates of prevalence in the weighted part II sample identical to those in the part I sample. The current analysis is based on the part II sample. More details about WMH sample design and weighting are presented elsewhere (Heeringa et al. 2008).

\section{Measures}

\section{Student status}

All respondents were asked if they were currently students or had finished their education and how many years of education they had completed. College students were defined as those in the age range of 18-22 years who had completed secondary school and were currently students (either part-time or full-time).
College students were compared with college attriters (i.e. respondents in the same age range who completed some college but did not graduate and were no longer students) and other non-students in the same age range with education less than or equal to a secondary school education.

\section{Mental disorders}

Mental disorders were assessed with version 3.0 of the Composite International Diagnostic Interview (CIDI; Kessler \& Ustun, 2004), a fully structured diagnostic interview administered by trained lay interviewers. DSM-IV criteria are used here. The CIDI assessed two lifetime mood disorders [major depressive disorder (MDD) and broadly defined bipolar disorder (BPD)]; including BPD-I, BPD-II and subthreshold $\mathrm{BPD}$, which was defined using criteria described elsewhere (Kessler et al. 2006), five lifetime anxiety disorders [separation anxiety disorder, panic disorder with or without agoraphobia, generalized anxiety disorder (GAD), phobia (either agoraphobia without a history of panic disorder, social phobia, or specific phobia), post-traumatic stress disorder (PTSD)], four disruptive behavior disorders [attention-deficit/hyperactivity disorder (ADHD), oppositional-defiant disorder, conduct disorder, intermittent explosive disorder] and four substance disorders (alcohol abuse with or without dependence; alcohol dependence with abuse, drug abuse with or without dependence, drug dependence with abuse). Age-of-onset of each disorder was assessed using special probing techniques shown experimentally to improve recall accuracy (Knäuper et al. 1999). Age-of-onset reports were used to determine whether each respondent had a history of each disorder prior to the typical age of beginning college (i.e. ages $0-17$ years). DSM-IV organic exclusion rules and diagnostic hierarchy rules were used other than for substance abuse, which was defined with or without dependence. As detailed elsewhere (Haro et al. 2006), generally good concordance was found between these CIDI diagnoses and blinded clinical diagnoses based on clinical reappraisal interviews with the SCID (First et al. 1994).

\section{Treatment of 12-month disorders}

Respondents who met criteria for any 12-month DSM-IV/CIDI disorder were presented with a list of mental health care providers (e.g. psychiatrist, psychologist, psychotherapist), general medical providers (e.g. general practitioner, cardiologist or other medical specialist, nurse), human services professionals (e.g. social workers, spiritual advisor), alternative therapists (e.g. herbalist, spiritualist), and self-help groups and asked if they ever sought help from each of these 
types of individuals or settings 'for problems with your emotions, nerves, or your use of alcohol or drugs', and, if so, how recently they did so. Respondents who reported seeking treatment at any time in the past 12 months were then asked how many visits they made in the past over that time period to each type of individual or setting. Consistent with earlier WMH analyses (Wang et al. 2007), we defined minimally adequate treatment as either (i) making at least four visits in the past 12 months to any type of treatment provider, (ii) making at least two visits and using any type of medication for their emotional problems, or (iii) still being in treatment at the time of interview.

\section{Analysis methods}

All analyses used weighted data. Cross-tabulations were used to estimate 12-month prevalence of each DSM-IV/CIDI disorder as of the time of interview and to decompose prevalence estimates into those with pre-matriculation onsets (i.e. ages $0-17$ years) and post-matriculation onsets (ages 18+ years), noting that the definition of pre-matriculation disorders was conservative in that virtually of those classified prematriculation did, in fact, start before college entry while some unknown number of disorders with onsets after the age of 17 years started before the respondent's college entry. These estimates were made separately for each disorder pooled across countries among college students, college attriters and respondents who had never entered college. A second set of crosstabulations was then estimated for lifetime prevalence of each disorder in the total sample separately among respondents in each of the same subsamples. Logistic regression analysis was used to estimate the odds ratio (OR) of 12-month and lifetime prevalence of each disorder within each of three pairs of subgroups: (i) students $v$. respondents who never entered college, (ii) attriters $v$. respondents who never entered college, and (iii) students $v$. attriters. Logistic regression coefficients and standard errors were exponentiated to produce ORs with 95\% confidence intervals (CIs). Logistic models included dummy control variables for surveys. We also evaluated significance of differences in ORs across countries at different income levels. Statistical significance was consistently evaluated using 0.05-level two-tailed tests. The design-based Taylor series method (Wolter, 1985) implemented in the SAS software system (SAS Institute Inc., 2010) was used to adjust significance tests for the weighting and clustering of observations. Overall fit of the logistic models was assessed by calculating area under the receiver operating characteristic curve (area under the curve; AUC).

\section{Results}

\section{Distribution of students}

A mean of 30.9 (S.E. $=0.9$ ) \% of respondents aged 18-22 years across surveys were students, with a range between 4.1\% (Shenzhen, PRC) and 59.0\% (BeijingShanghai, PRC and Medellín, Colombia) Aggregate rates were similar for males $(32.0 \%)$ and females (29.7\%). See online Supplementary material.

\section{What is the 12-month prevalence of DSM-IV/CIDI disorders among college students?}

The 12-month prevalence of any DSM-IV/CIDI disorder was $20.3 \%$ among college students compared with $25.0 \%$ among attriters and $21.4 \%$ among other non-students (Table 1). Students also had a lower mean number of overall disorders (23.9/100 persons) than either attriters (39.7/100) or other non-students (32.8/100). Anxiety disorders were the most prevalent class of disorders across all groups in the full sample (11.7-14.7\%) followed by mood disorders (6.0-9.9\%), substance disorders $(4.5-6.7 \%)$ and behavioral disorders $(2.8-5.3 \%)$. The most prevalent individual disorders were phobias (9.0-11.1\%) and MDD (4.5-7.7\%). Logistic models controlling age-sex and survey found that students had significantly higher prevalence than attriters of panic disorder and lower prevalence of behavioral disorders, while students had significantly lower prevalence than other non-students of quite a few disorders (GAD, PTSD, any mood disorder, ADHD, oppositional-defiant disorder, drug dependence) as well as of number of disorders.

\section{What proportion of 12-month DSM-IV/CIDI disorders had onsets prior to college entry?}

The vast majority $(83.1 \%)$ of students with 12 -month disorders reported pre-matriculation onsets (Table 2). The only disorder-specific exceptions were that postmatriculation onsets were more common than prematriculation onsets among students for 12-month panic disorder (51.8\%) and alcohol abuse-dependence (70.0-67.6\%) and that post-matriculation onsets characterized sizable minorities of students with 12-month GAD (38.4\%), PTSD (41.3\%), mood disorders (41.6\%) and drug abuse $(46.2 \%)$. Relative proportions of pre$v$. post-matriculation onsets were generally comparable among attriters and other non-students as among students $\left(\chi_{1}^{2}=0.0-3.4, p=0.92-0.06\right)$. The two exceptions to this general pattern were that a significantly higher proportion of students $(70.0 \%)$ than attriters $\left(42.5 \% ; \chi_{1}^{2}=8.6, p=0.003\right)$ with 12 -month alcohol abuse had post-matriculation onsets and a significantly higher proportion of students $(16.9 \%)$ than nonstudents $\left(12.3 \% ; \chi_{1}^{2}=5.4, p=0.020\right)$ with any 12 -month 
Table 1. Pooled 12-month prevalence of DSM-IV/CIDI mental disorders separately among respondents aged 18-22 years who were current students, college attriters and non-students in the same age range ${ }^{\text {a }}$

\begin{tabular}{|c|c|c|c|c|c|c|}
\hline & $\begin{array}{l}\text { Students } \\
\% \text { (s.E.) }\end{array}$ & $\begin{array}{l}\text { Attriters } \\
\% \text { (s.E.) }\end{array}$ & $\begin{array}{l}\text { Other } \\
\% \text { (s.E.) }\end{array}$ & $\begin{array}{l}\text { Students } v \text {. attriters }{ }^{\mathrm{b}} \\
\text { OR }(95 \% \mathrm{CI})\end{array}$ & $\begin{array}{l}\text { Students } v \text {. other } \\
\text { OR }(95 \% \text { CI })\end{array}$ & $\mathrm{AUC}^{\mathrm{C}}$ \\
\hline \multicolumn{7}{|l|}{ I. Anxiety disorders } \\
\hline Separation anxiety disorder & $1.3(0.7)$ & $1.5(0.5)$ & $1.2(0.2)$ & $1.2(0.3-3.8)$ & $1.1(0.3-3.6)$ & 0.84 \\
\hline Panic disorder & $1.2(0.3)$ & $1.1(0.3)$ & $1.4(0.2)$ & $2.6(1.1-5.9)^{*}$ & $0.9(0.5-1.5)$ & 0.72 \\
\hline GAD & $0.4(0.1)$ & $0.8(0.3)$ & $0.8(0.1)$ & $0.9(0.3-2.5)$ & $0.5(0.3-1.0)^{*}$ & 0.70 \\
\hline Any phobia & $9.0(1.1)$ & $11.1(1.5)$ & $9.9(0.6)$ & $0.9(0.6-1.3)$ & $0.9(0.7-1.2)$ & 0.68 \\
\hline PTSD & $1.3(0.3)$ & $2.7(0.6)$ & $2.0(0.3)$ & $0.9(0.5-1.7)$ & $0.6(0.3-1.0)^{*}$ & 0.83 \\
\hline Any & $11.7(1.3)$ & $14.7(1.6)$ & $12.9(0.6)$ & $1.0(0.7-1.4)$ & $0.9(0.7-1.2)$ & 0.69 \\
\hline \multicolumn{7}{|l|}{ II. Mood disorders } \\
\hline MDD & $4.5(0.5)$ & $7.7(0.9)$ & $5.1(0.4)$ & $0.7(0.5-1.1)$ & $0.9(0.6-1.2)$ & 0.67 \\
\hline Bipolar & $1.8(0.4)$ & $2.3(0.5)$ & $2.6(0.3)$ & $1.1(0.6-2.2)$ & $0.6(0.4-1.0)$ & 0.75 \\
\hline Any & $6.0(0.7)$ & $9.9(1.0)$ & $7.6(0.5)$ & $0.8(0.6-1.1)$ & $0.7(0.6-1.0)^{*}$ & 0.68 \\
\hline \multicolumn{7}{|l|}{ III. Behavioral disorders } \\
\hline ADHD & $0.4(0.2)$ & $1.5(0.7)$ & $1.0(0.1)$ & $0.4(0.1-1.4)$ & $0.3(0.1-0.9)^{*}$ & 0.83 \\
\hline Conduct disorder & $0.2(0.1)$ & $0.4(0.3)$ & $0.5(0.1)$ & $0.4(0.0-4.3)$ & $0.3(0.1-1.4)$ & 0.86 \\
\hline ODD & $0.2(0.1)$ & $1.0(0.5)$ & $0.6(0.1)$ & $0.3(0.1-1.6)$ & $0.3(0.1-0.9)^{*}$ & 0.82 \\
\hline IED & $2.4(0.4)$ & $3.0(0.7)$ & $2.4(0.3)$ & $0.8(0.5-1.3)$ & $0.9(0.6-1.3)$ & 0.81 \\
\hline Any & $2.8(0.4)$ & $5.3(1.1)$ & $3.8(0.3)$ & $0.6(0.4-1.0)^{*}$ & $0.7(0.5-0.9)^{*}$ & 0.79 \\
\hline \multicolumn{7}{|l|}{ IV. Substance disorders } \\
\hline Alcohol abuse & $2.5(0.4)$ & $4.2(0.9)$ & $3.1(0.3)$ & $0.7(0.4-1.3)$ & $0.7(0.5-1.0)$ & 0.77 \\
\hline Alcohol dependence & $1.4(0.4)$ & $1.6(0.5)$ & $1.5(0.2)$ & $1.1(0.5-2.7)$ & $0.8(0.4-1.5)$ & 0.77 \\
\hline Drug abuse & $0.7(0.2)$ & $1.3(0.5)$ & $1.0(0.2)$ & $0.7(0.2-1.9)$ & $0.6(0.3-1.4)$ & 0.82 \\
\hline Drug dependence & $0.2(0.1)$ & $1.1(0.4)$ & $0.9(0.2)$ & $0.3(0.1-1.2)$ & $0.2(0.1-0.8)^{*}$ & 0.83 \\
\hline Any & $4.5(0.6)$ & $6.7(1.1)$ & $5.8(0.4)$ & $0.9(0.5-1.4)$ & $0.7(0.5-0.9)^{*}$ & 0.78 \\
\hline \multicolumn{7}{|l|}{ V. Total disorders } \\
\hline Low/lower middle-income countries & $12.8(1.9)$ & $13.4(2.5)$ & $14.7(1.1)$ & $1.1(0.6-1.9)$ & $0.8(0.6-1.2)$ & 0.64 \\
\hline Upper-middle-income countries & $21.8(4.9)$ & $31.8(6.5)$ & $21.9(1.9)$ & $0.8(0.3-2.0)$ & $1.0(0.5-1.8)$ & 0.63 \\
\hline High-income countries & $25.2(1.7)$ & $27.5(2.6)$ & $27.3(1.4)$ & $1.1(0.8-1.5)$ & $0.9(0.7-1.1)$ & 0.66 \\
\hline Total & $20.3(1.4)$ & $25.0(2.0)$ & $21.4(0.8)$ & $1.0(0.8-1.3)$ & $0.9(0.7-1.1)$ & 0.67 \\
\hline$n$ & 1572 & 702 & 3476 & - & - & - \\
\hline
\end{tabular}

DSM-IV, Diagnostic and Statistical Manual of Mental Disorders, 4th edition; CIDI, Composite International Diagnostic Interview; S.E., standard error; OR, odds ratio; CI, confidence interval; AUC, area under the receiver operating characteristic curve; GAD, generalized anxiety disorder; PTSD, post-traumatic stress disorder; MDD, major depressive disorder; ADHD, attention-deficit/hyperactivity disorder; ODD, oppositional-defiant disorder; IED, intermittent explosive disorder.

${ }^{\text {a }}$ Respondents were limited to those in the part II sample who were 18-22 years old at the time of interview and had not graduated from college.

${ }^{\mathrm{b}}$ Based on a pooled within-survey logistic regression model adjusting for between-survey variation in the association of age-sex with student status.

${ }^{\mathrm{c}}$ The AUCs were generated from logistic models in which dummy variables for being an attriter or other non-student were included as predictors of the mental disorder in the row heading or, in the case of part VI, number of disorders estimated in an order logistic framework, controlling age-sex and survey.

* Significant $(p<0.05$, two-sided test).

disorders had at least one such disorder with a postmatriculation onset.

\section{Do pre-matriculation-onset DSM-IV/CIDI disorders predict college entry?}

Somewhat smaller proportions of respondents who entered college $(25.3 \%)$ than those who did not $(27.2 \%$;
$28.2 \%$ of those who graduated from secondary school) met criteria for at least one lifetime pre-matriculation DSM-IV/CIDI disorder (Table 3). The mean number of disorders was also lower for students (36.2/100) than non-students (48.9-46.4/100). Anxiety disorders were consistently much more common across these samples (15.7-19.0\%) than either mood (7.9-8.3\%), behavioral 
Table 2. Pooled percentages of 12-month DSM-IV/CIDI disorders with pre-matriculation onsets ${ }^{\mathrm{a}}$ separately among respondents aged 18-22 years who were current students, college attriters and non-students ${ }^{\mathrm{b}}$ in the same age range ${ }^{\mathrm{c}}$

\begin{tabular}{|c|c|c|c|c|c|}
\hline & $\begin{array}{l}\text { Students } \\
\% \text { (S.E.) }\end{array}$ & $\begin{array}{l}\text { Attriters } \\
\% \text { (S.E.) }\end{array}$ & $\begin{array}{l}\text { Other }^{\mathrm{d}} \\
\% \text { (S.E.) }\end{array}$ & $\begin{array}{l}\text { Students } v \text {. } \\
\text { attriters } \\
\chi^{2}\end{array}$ & $\begin{array}{l}\text { Students } v \text {. } \\
\text { other } \\
\chi^{2}\end{array}$ \\
\hline \multicolumn{6}{|l|}{ I. Anxiety disorders } \\
\hline Separation anxiety disorder & $70.4(18.4)$ & $44.9(17.5)$ & $62.6(7.0)$ & 0.3 & 2.2 \\
\hline Panic disorder & $48.2(12.1)$ & $75.6(13.4)$ & $84.6(4.3)$ & 0.1 & 0.7 \\
\hline GAD & $61.6(12.6)$ & $48.5(17.4)$ & $76.9(6.5)$ & 0.4 & 2.3 \\
\hline Any phobia & $95.6(1.5)$ & $89.8(3.6)$ & $97.6(0.9)$ & 0.2 & 1.2 \\
\hline PTSD & $58.7(11.2)$ & $73.8(9.3)$ & $77.4(5.2)$ & 0.0 & 1.5 \\
\hline Any & $91.6(1.8)$ & $86.9(3.1)$ & $93.8(1.1)$ & 0.0 & 1.5 \\
\hline \multicolumn{6}{|l|}{ II. Mood disorders } \\
\hline MDD & $53.7(6.2)$ & $63.5(6.3)$ & $64.9(3.4)$ & 0.5 & 2.9 \\
\hline Bipolar & $61.4(10.8)$ & $58.6(11.1)$ & $61.8(4.9)$ & 0.5 & 0.1 \\
\hline Any & $58.4(5.3)$ & $64.4(5.5)$ & $63.9(2.8)$ & 0.6 & 0.4 \\
\hline \multicolumn{6}{|l|}{ III. Behavior disorders } \\
\hline ADHD & $100.0(0.0)$ & $100.0(0.0)$ & $100.0(0.0)$ & - & - \\
\hline Conduct disorder & $100.0(0.0)$ & $100.0(0.0)$ & $100.0(0.0)$ & - & - \\
\hline ODD & $100.0(0.0)$ & $100.0(0.0)$ & $99.0(1.0)$ & - & - \\
\hline IED & $90.5(5.7)$ & $84.3(7.5)$ & $88.2(2.8)$ & 0.1 & 0.0 \\
\hline Any & $92.1(4.8)$ & $92.0(4.3)$ & $94.2(1.6)$ & 0.0 & 0.1 \\
\hline \multicolumn{6}{|l|}{ IV. Substance disorders } \\
\hline Alcohol abuse & $30.0(6.0)$ & $57.5(11.9)$ & $43.6(4.9)$ & $8.6^{*}$ & 3.4 \\
\hline Alcohol dependence & $32.4(13.7)$ & $32.3(12.9)$ & $45.5(6.3)$ & 0.0 & 0.1 \\
\hline Drug abuse & $53.8(15.7)$ & $52.4(19.8)$ & $61.7(7.9)$ & 1.5 & 1.3 \\
\hline Drug dependence & $100.0(0.0)$ & $66.4(17.3)$ & $60.8(8.5)$ & - & - \\
\hline Any & $46.6(6.7)$ & $61.2(8.5)$ & $55.8(3.6)$ & 3.3 & 0.9 \\
\hline \multicolumn{6}{|l|}{ V. Total disorders } \\
\hline Any & $83.1(2.3)$ & $83.6(3.0)$ & $87.7(1.2)$ & 0.9 & $5.4^{*}$ \\
\hline
\end{tabular}

DSM-IV, Diagnostic and Statistical Manual of Mental Disorders, 4th edition; CIDI, Composite International Diagnostic Interview; S.E., standard error; GAD, generalized anxiety disorder; PTSD, post-traumatic stress disorder; MDD, major depressive disorder; $\mathrm{ADHD}$, attention-deficit/hyperactivity disorder; ODD, oppositional-defiant disorder; IED, intermittent explosive disorder.

${ }^{\text {a }}$ Pre-matriculation-onset disorders were defined as those with onsets at ages $0-17$ years.

${ }^{\mathrm{b}}$ Post-matriculation-onset disorders were defined as those with onsets at ages $18+$ years.

${ }^{\mathrm{c}}$ Respondents were limited to those in the part II sample who were 18-22 years old at the time of interview and had not graduated from college.

d 'All other' respondents were defined as those who never entered college whether or not they graduated from secondary school.

* Significant $(p<0.05)$.

(6.8-7.8\%) or substance (5.0-6.9\%) disorders. Phobia was by far the most prevalent individual disorder (11.6$13.9 \%$ ) followed by MDD (5.8-6.2\%). Logistic regression analysis controlling age-sex and survey found that history of one or more pre-matriculation disorders was associated with significantly reduced odds of subsequent matriculation among respondents who graduated from secondary school (OR 0.8, 95\% CI 0.7-1.0), although further analysis (see online Supplementary material) showed that this was true only in high-income countries (OR 0.7, 95\% CI 0.6-0.9). A similar pattern was found for a number of disorders. Disaggregated analysis showed that a number of individual anxiety (separation anxiety disorder, panic disorder, PTSD), behavioral (conduct disorder, oppositional-defiant disorder) and substance (alcohol and drug abuse) disorders were implicated in these aggregate associations, with mood disorders the only class of disorders not significant in any of the comparisons. Replication of these analyses separately among males and females (see online Supplementary material) revealed that the significant inverse associations between pre-matriculation disorders and subsequent matriculation were for the most part stronger among females than males. 
Table 3. Pooled lifetime prevalence of pre-matriculation-onset DSM-IV/CIDI mental disorders ${ }^{\text {a }}$ separately among respondents aged 18-22 years who entered college (both current students and attriters), secondary school graduates in the same age range who never entered college and all other respondents ${ }^{\mathrm{b}}$ in the same age range $\mathrm{c}^{\mathrm{c}}$

\begin{tabular}{|c|c|c|c|c|c|c|c|}
\hline & \multirow{3}{*}{$\begin{array}{l}\text { College } \\
\text { entrants } \\
\% \text { (s.E.) }\end{array}$} & \multicolumn{2}{|c|}{$\begin{array}{l}\text { Other respondents who } \\
\text { were secondary school... }\end{array}$} & \multirow{2}{*}{\multicolumn{2}{|c|}{$\begin{array}{l}\text { College entrants } v . \\
\text { graduates }{ }^{\mathrm{e}}\end{array}$}} & \multirow{2}{*}{\multicolumn{2}{|c|}{$\begin{array}{l}\text { College entrants } v \text {. } \\
\text { total }^{\mathrm{e}}\end{array}$}} \\
\hline & & \multirow{2}{*}{$\frac{\text { Graduates }}{\% \text { (S.E.) }}$} & \multirow{2}{*}{$\begin{array}{l}\text { Total }^{\mathrm{d}} \\
\% \text { (S.E.) }\end{array}$} & & & & \\
\hline & & & & OR $(95 \% \mathrm{CI})$ & AUC & OR $(95 \% \mathrm{CI})$ & AUC \\
\hline \multicolumn{8}{|l|}{ I. Anxiety disorders } \\
\hline Separation anxiety disorder & $2.4(0.3)$ & $4.2(0.5)$ & $4.0(0.4)$ & $0.6(0.3-1.1)$ & 0.85 & $0.5(0.3-0.9)^{*}$ & 0.85 \\
\hline Panic disorder & $1.1(0.2)$ & $1.4(0.2)$ & $1.4(0.2)$ & $0.6(0.3-1.0)^{*}$ & 0.70 & $0.6(0.3-1.1)$ & 0.69 \\
\hline GAD & $0.9(0.2)$ & $1.2(0.2)$ & $1.2(0.2)$ & $0.6(0.3-1.2)$ & 0.75 & $0.6(0.3-1.1)$ & 0.75 \\
\hline Any phobia & $11.6(0.7)$ & $13.9(0.8)$ & $12.5(0.6)$ & $0.8(0.6-1.0)$ & 0.66 & $0.9(0.7-1.1)$ & 0.67 \\
\hline PTSD & $1.9(0.3)$ & $2.7(0.4)$ & $2.4(0.3)$ & $0.6(0.3-1.0)^{*}$ & 0.83 & $0.6(0.4-0.9)^{*}$ & 0.83 \\
\hline Any & $15.7(0.8)$ & $19.0(1.0)$ & $17.3(0.7)$ & $0.8(0.6-1.0)^{*}$ & 0.69 & $0.8(0.7-1.0)$ & 0.69 \\
\hline \multicolumn{8}{|l|}{ II. Mood disorders } \\
\hline MDD & $5.9(0.5)$ & $6.2(0.4)$ & $5.8(0.4)$ & $0.9(0.6-1.1)$ & 0.69 & $0.8(0.6-1.1)$ & 0.69 \\
\hline Bipolar & $2.1(0.3)$ & $2.3(0.4)$ & $2.4(0.3)$ & $0.8(0.5-1.4)$ & 0.74 & $0.8(0.5-1.2)$ & 0.74 \\
\hline Any & $7.9(0.5)$ & $8.3(0.6)$ & $8.1(0.5)$ & $0.8(0.6-1.1)$ & 0.70 & $0.8(0.6-1.0)$ & 0.70 \\
\hline \multicolumn{8}{|l|}{ III. Behavioral disorders } \\
\hline ADHD & $2.1(0.3)$ & $2.0(0.4)$ & $2.0(0.3)$ & $1.1(0.6-1.8)$ & 0.85 & $0.8(0.5-1.2)$ & 0.84 \\
\hline Conduct disorder & $1.6(0.3)$ & $2.9(0.4)$ & $2.8(0.3)$ & $0.5(0.3-0.8)^{*}$ & 0.88 & $0.4(0.2-0.6)^{*}$ & 0.88 \\
\hline ODD & $2.3(0.3)$ & $3.1(0.4)$ & $2.9(0.3)$ & $0.7(0.4-1.2)$ & 0.86 & $0.6(0.4-0.9)^{*}$ & 0.85 \\
\hline IED & $3.1(0.5)$ & $2.6(0.3)$ & $2.7(0.3)$ & $1.3(0.9-1.9)$ & 0.83 & $1.0(0.8-1.4)$ & 0.83 \\
\hline Any & $6.8(0.6)$ & $7.8(0.7)$ & $7.5(0.5)$ & $0.9(0.6-1.2)$ & 0.83 & $0.7(0.6-0.9)^{*}$ & 0.82 \\
\hline \multicolumn{8}{|l|}{ IV. Substance disorders } \\
\hline Alcohol abuse & $2.4(0.3)$ & $3.5(0.4)$ & $4.0(0.4)$ & $0.6(0.4-0.9)^{*}$ & 0.78 & $0.4(0.3-0.6)^{*}$ & 0.78 \\
\hline Alcohol dependence & $0.8(0.2)$ & $0.8(0.2)$ & $1.3(0.2)$ & $1.0(0.5-2.0)$ & 0.82 & $0.6(0.3-1.1)$ & 0.82 \\
\hline Drug abuse & $1.7(0.3)$ & $1.9(0.3)$ & $2.3(0.3)$ & $0.7(0.4-1.2)$ & 0.83 & $0.6(0.4-0.9)^{*}$ & 0.82 \\
\hline Drug dependence & $1.0(0.2)$ & $1.0(0.2)$ & $1.2(0.2)$ & $0.9(0.4-1.8)$ & 0.81 & $0.7(0.4-1.2)$ & 0.80 \\
\hline Any & $5.0(0.5)$ & $5.7(0.5)$ & $6.9(0.4)$ & $0.7(0.5-1.0)^{*}$ & 0.80 & $0.5(0.4-0.7)^{*}$ & 0.80 \\
\hline \multicolumn{8}{|l|}{ V. Total disorders } \\
\hline Low/lower middle-income countries & $15.8(1.8)$ & $19.9(2.0)$ & $16.5(1.2)$ & $0.8(0.5-1.1)$ & 0.67 & $0.9(0.6-1.3)$ & 0.67 \\
\hline Upper-middle-income countries & $29.5(4.5)$ & $28.9(3.5)$ & $29.1(2.1)$ & $1.0(0.6-1.9)$ & 0.63 & $0.9(0.5-1.4)$ & 0.63 \\
\hline High-income countries & $29.3(1.6)$ & $33.2(1.8)$ & $35.7(1.4)$ & $0.7(0.7-1.0)^{*}$ & 0.70 & $0.7(0.5-0.8)^{*}$ & 0.70 \\
\hline Total & $25.3(1.0)$ & $28.2(1.1)$ & $27.2(0.9)$ & $0.8(0.7-1.0)^{*}$ & 0.70 & $0.8(0.6-0.9)^{*}$ & 0.70 \\
\hline$n$ & 2274 & 1571 & 3476 & - & & - & \\
\hline
\end{tabular}

DSM-IV, Diagnostic and Statistical Manual of Mental Disorders, 4th edition; CIDI, Composite International Diagnostic Interview; S.E., standard error; OR, odds ratio; CI, confidence interval; AUC, area under the receiver operating characteristic curve; GAD, generalized anxiety disorder; PTSD, post-traumatic stress disorder; MDD, major depressive disorder; ADHD, attention-deficit/hyperactivity disorder; ODD, oppositional-defiant disorder; IED, intermittent explosive disorder.

a Pre-matriculation-onset disorders were defined as those with onsets at ages 0-17 years.

b 'All other' respondents were defined as those who never entered college whether or not graduated from secondary school.

${ }^{\mathrm{c}}$ Respondents were limited to those in the part II sample who were 18-22 years old at the time of interview and had not graduated from college.

${ }^{\mathrm{d}}$ Secondary school graduates or non-graduates.

${ }^{\mathrm{e}}$ Based on a single pooled logistic model for each row in which separate dummy variables for being an attriter or other non-student were included as predictors of the mental disorder in the row heading or, in the case of part VI, number of disorders estimated in an ordered logistic framework, controlling age-sex and survey.

* Significant $(p<0.05$, two-sided test). 


\section{Do pre- and post-matriculation-onset DSM-IV/CIDI disorders predict college attrition?}

A significantly lower proportion of students than attriters had one or more pre-matriculation-onset mental disorders $(22.9 \%$ v. 30.3\%; $t=2.7, p=0.007)$. This pattern is consistent with pre-matriculation disorders predicting subsequent attrition among college students, although this difference became non-significant in the logistic regression analysis controlling age-sex and survey (OR 0.9, 95\% CI 0.6-1.1) (Table 4). However, a comparable model that looked at number of prematriculation disorders $(0.1,2.3,4+)$ found a significantly lower odds of being a student than attriter (OR 0.8, 95\% CI 0.6-1.0). The individual disorders significantly implicated in this association were all substance disorders. The association was significant only in high-income countries. Replication of these analyses separately among males and females (see online Supplementary material) showed that pre-matriculation alcohol and drug abuse predicted attrition among makes (with ORs of 0.2-0.4), while MDD, alcohol abuse and drug dependence were significant among females (with ORs of 0.2-0.6).

A significantly lower proportion of students than attriters also had one or more post-matriculation-onset disorders $(6.7 \%$ v. 10.9\%; $t=2.5, p=0.006)$, but again this difference became non-significant in the logistic regression analysis (OR 1.2, 95\% CI 0.8-1.8). A parallel model for number of disorders was also insignificant. Logistic regression found only one individual disorder, panic disorder, with significantly different odds of post-matriculation onset among attriters compared with students, but in this case the odds were significantly higher, not lower, among students than attriters (OR 5.0, 95\% CI 1.1-22.1). Replication separately among males and females (see online Supplementary material) showed that the pattern differed by sex, with the significantly elevated odds of postmatriculation panic disorder among students compared with attriters confined to males and the only significant OR among females involving postmatriculation onset GAD being lower among students than attriters (OR 0.2, 95\% CI 0.1-0.5).

\section{What proportion of students with 12-month DSM-IV/CIDI disorders receive treatment?}

Roughly one-sixth (16.4\%) of students with 12-month DSM-IV/CIDI disorders received minimally adequate treatment for these disorders in the 12 months before interview (Table 5). This treatment rate was not significantly different from that of attriters $\left(16.6 \% ; \chi_{1}^{2}=0.8, p\right.$ $=0.37)$ or other non-students $\left(10.6 \% ; \chi_{1}^{2}=2.8, p=0.09\right)$. However, the treatment rate of students decreased monotonically with country income level, from $23.1 \%$ in high-income countries, to $11.4 \%$ in upper-middleand $6.7 \%$ in lower-middle/low-income countries $\left(\chi_{2}^{2}=\right.$ $7.4, p=0.025)$.

\section{Discussion}

We presented here the first large-scale cross-national investigation of 12-month and lifetime prevalence of mental disorders among college students, the associations of pre- and post-matriculation mental disorders with college matriculation and attrition, and 12-month treatment of mental disorders among college students. The results showed that a substantial proportion of college students have 12-month DSM-IV/CIDI disorders, that the vast majority of cases had prematriculation onsets, that pre-matriculation disorders are associated with both reduced odds of college matriculation and elevated odds of attrition, and that only a small minority of college students receive even minimally adequate treatment for their mental disorders.

In considering intervention possibilities, an important question unanswered by our results is whether the pre-matriculation disorders we found to be associated with attrition are stable. This question arises because we made a great many comparisons in the analyses, leading to the possibility that some of the associations judged to be significant were false positives. Replication is needed to evaluate this issue. This replication would ideally be carried out in a prospective sample that assessed students at the time of college entry and followed them through their college careers to evaluate the importance of pre-matriculation predictors of college outcomes.

Assuming that this replication supports our finding that pre-matriculation mental disorders are associated with attrition, a second important unanswered question is whether these disorders are causal risk factors or only risk markers of attrition (Kraemer et al. 1997). Our findings that pre-matriculation substance disorders (males and females) and MDD (females) predicted subsequent attrition were also found in previous epidemiological studies (Kessler et al. 1995; Eisenberg et al. 2009; Hunt et al. 2010; Mojtabai et al. 2015), but none of these studies provided any evidence that these disorders are causes rather than non-causal correlates. It is plausible to think that the associations are non-causal because risk factors for adolescent substance (Kilpatrick et al. 2000) and mood (Lewinsohn et al. 1998; Costello et al. 2002) disorders have many similarities with risk factors for college attrition (Ishitani \& DesJardins, 2002; Stratton et al. 2006; Hartley, 2010), such as high stress, interpersonal discord and diminished social support, none of which we controlled in our analyses. To the extent that 
Table 4. Pooled lifetime prevalence of pre-matriculation ${ }^{\mathrm{a}}$ - and post-matriculation ${ }^{\mathrm{b}}$-onset DSM-IV/CIDI mental disorders separately among respondents aged 18-22 years who were current students or college attriters ${ }^{\mathrm{c}}$

\begin{tabular}{|c|c|c|c|c|c|c|c|c|}
\hline & \multirow{2}{*}{\multicolumn{2}{|c|}{ Students }} & \multirow{2}{*}{\multicolumn{2}{|c|}{ Attriters }} & \multicolumn{4}{|c|}{ Students $v$. attriters } \\
\hline & & & & & \multicolumn{2}{|l|}{ Pre- ${ }^{\mathrm{e}}$} & \multicolumn{2}{|l|}{ Post $-{ }^{\mathrm{f}}$} \\
\hline & \multirow{2}{*}{$\begin{array}{l}\text { Pre- } \\
\% \text { (S.E.) }\end{array}$} & \multirow{2}{*}{$\begin{array}{l}\text { Post- }^{\mathrm{d}} \\
\% \text { (S.E.) }\end{array}$} & \multirow{2}{*}{$\begin{array}{l}\text { Pre- } \\
\% \text { (S.E.) }\end{array}$} & \multirow{2}{*}{$\begin{array}{l}\text { Post- }^{\mathrm{d}} \\
\% \text { (S.E.) }\end{array}$} & & 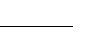 & & 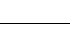 \\
\hline & & & & & OR $(95 \% \mathrm{CI})$ & AUC & OR $(95 \%$ CI $)$ & AUC \\
\hline \multicolumn{9}{|l|}{ I. Anxiety disorders } \\
\hline Separation anxiety disorder & $2.7(0.8)$ & $0.7(0.2)$ & $1.8(0.5)$ & $1.1(0.3)$ & $1.5(0.6-3.5)$ & 0.85 & $1.2(0.6-2.5)$ & 0.89 \\
\hline Panic disorder & $1.1(0.3)$ & $0.6(0.3)$ & $1.1(0.3)$ & $0.3(0.2)$ & $1.8(0.8-3.9)$ & 0.69 & $5.0(1.1-22.1)^{*}$ & 0.82 \\
\hline GAD & $0.7(0.2)$ & $0.2(0.1)$ & $1.3(0.5)$ & $0.9(0.3)$ & $0.7(0.3-1.9)$ & 0.75 & $0.6(0.2-1.4)$ & 0.79 \\
\hline Any phobia & $11.3(1.1)$ & $0.5(0.1)$ & $12.3(1.5)$ & $1.3(0.5)$ & $1.0(0.7-1.4)$ & 0.67 & $0.7(0.2-2.6)$ & 0.74 \\
\hline PTSD & $1.1(0.3)$ & $0.6(0.1)$ & $3.6(0.7)$ & $0.7(0.3)$ & $0.5(0.3-1.1)$ & 0.83 & $2.0(0.8-4.8)$ & 0.83 \\
\hline Any & $15.1(1.4)$ & $1.5(0.2)$ & $16.9(1.7)$ & $2.8(0.6)$ & $1.0(0.8-1.4)$ & 0.69 & $1.1(0.6-2.0)$ & 0.77 \\
\hline \multicolumn{9}{|l|}{ II. Mood disorders } \\
\hline MDD & $4.8(0.5)$ & $3.4(0.5)$ & $8.3(1.1)$ & $4.0(0.8)$ & $0.7(0.5-1.1)$ & 0.69 & $1.4(0.8-2.3)$ & 0.72 \\
\hline Bipolar & $1.9(0.4)$ & $0.8(0.3)$ & $2.6(0.6)$ & $1.2(0.4)$ & $0.8(0.4-1.5)$ & 0.74 & $1.2(0.4-3.2)$ & 0.79 \\
\hline Any & $6.6(0.6)$ & $3.9(0.6)$ & $10.8(1.3)$ & $5.2(0.9)$ & $0.7(0.5-1.1)$ & 0.70 & $1.3(0.8-2.0)$ & 0.72 \\
\hline \multicolumn{9}{|l|}{ III. Behavioral disorders } \\
\hline ADHD & $1.9(0.4)$ & $0.0(0.0)$ & $2.6(0.7)$ & $0.0(0.0)$ & $1.2(0.5-2.6)$ & 0.84 & _g & - \\
\hline Conduct disorder & $1.2(0.3)$ & $0.0(0.0)$ & $2.4(0.7)$ & $0.0(0.0)$ & $0.8(0.3-1.7)$ & 0.88 & $-^{g}$ & - \\
\hline ODD & $1.9(0.4)$ & $0.0(0.0)$ & $3.3(0.7)$ & $0.0(0.0)$ & $0.8(0.4-1.5)$ & 0.85 & $-^{g}$ & - \\
\hline IED & $2.9(0.5)$ & $0.2(0.1)$ & $3.7(0.8)$ & $0.5(0.2)$ & $0.8(0.5-1.4)$ & 0.83 & $0.8(0.2-3.7)$ & 0.88 \\
\hline Any & $5.9(0.7)$ & $0.2(0.2)$ & $8.7(1.2)$ & $0.5(0.3)$ & $0.9(0.6-1.3)$ & 0.82 & $0.8(0.2-4.6)$ & 0.88 \\
\hline \multicolumn{9}{|l|}{ IV. Substance disorders } \\
\hline Alcohol abuse & $1.5(0.2)$ & $2.6(0.4)$ & $4.5(0.9)$ & $5.6(1.1)$ & $0.4(0.2-0.7)^{*}$ & 0.78 & $0.9(0.5-1.5)$ & 0.79 \\
\hline Alcohol dependence & $0.6(0.2)$ & $1.1(0.3)$ & $1.4(0.4)$ & $1.7(0.6)$ & $0.4(0.1-1.0)$ & 0.83 & $1.3(0.5-3.2)$ & 0.79 \\
\hline Drug abuse & $0.9(0.3)$ & $0.4(0.1)$ & $3.4(0.7)$ & $1.2(0.5)$ & $0.3(0.1-0.6)^{*}$ & 0.84 & $0.8(0.3-2.1)$ & 0.77 \\
\hline Drug dependence & $0.4(0.2)$ & $0.1(0.1)$ & $2.2(0.6)$ & $0.6(0.3)$ & $0.2(0.1-0.6)^{*}$ & 0.83 & $0.5(0.1-2.3)$ & 0.81 \\
\hline
\end{tabular}




\begin{tabular}{|c|c|c|c|c|c|c|c|c|}
\hline & \multirow{2}{*}{\multicolumn{2}{|c|}{ Students }} & \multirow{2}{*}{\multicolumn{2}{|c|}{ Attriters }} & \multicolumn{4}{|c|}{ Students $v$. attriters } \\
\hline & & & & & \multicolumn{2}{|l|}{ Pre ${ }^{e}$} & \multirow{2}{*}{\multicolumn{2}{|c|}{ Post- ${ }^{\mathrm{f}}$}} \\
\hline & \multirow{2}{*}{$\begin{array}{l}\text { Pre- } \\
\% \text { (s.E.) }\end{array}$} & \multirow{2}{*}{$\begin{array}{l}\text { Post- }^{\mathrm{d}} \\
\% \text { (S.E.) }\end{array}$} & \multirow{2}{*}{$\begin{array}{l}\text { Pre- } \\
\% \text { (S.E.) }\end{array}$} & \multirow{2}{*}{$\begin{array}{l}\text { Post- }^{\mathrm{d}} \\
\% \text { (S.E.) }\end{array}$} & & - & & \\
\hline & & & & & OR (95\% CI) & AUC & OR $(95 \% \mathrm{CI})$ & AUC \\
\hline Any & $3.2(0.5)$ & $3.5(0.5)$ & $8.8(1.1)$ & $7.7(1.2)$ & $0.4(0.3-0.6)^{*}$ & 0.80 & $0.9(0.5-1.5)$ & 0.79 \\
\hline \multicolumn{9}{|l|}{ V. Total disorders } \\
\hline Low/lower middle-income countries & $15.4(2.2)$ & $4.8(1.3)$ & $17.3(2.9)$ & $6.9(2.6)$ & $0.9(0.5-1.6)$ & 0.67 & $1.2(0.4-3.7)$ & 0.74 \\
\hline Upper-middle-income countries & $26.8(5.7)$ & $2.3(0.9)$ & $38.8(6.5)$ & $9.4(4.1)$ & $0.8(0.3-1.9)$ & 0.63 & $0.6(0.1-2.4)$ & 0.72 \\
\hline High-income countries & $26.9(1.8)$ & $10.3(1.3)$ & $32.9(2.8)$ & $12.8(2.1)$ & $0.8(0.6-1.1)$ & 0.70 & $1.3(0.8-2.1)$ & 0.70 \\
\hline Total & $22.9(1.6)$ & $6.7(0.7)$ & $30.3(2.1)$ & $10.9(1.5)$ & $0.9(0.6-1.1)$ & 0.70 & $1.2(0.8-1.8)$ & 0.73 \\
\hline$n$ & 1572 & $-^{\mathrm{h}}$ & 702 & $-{ }^{\mathrm{i}}$ & & & & \\
\hline
\end{tabular}

DSM-IV, Diagnostic and Statistical Manual of Mental Disorders, 4th edition; CIDI, Composite International Diagnostic Interview; S.E., standard error; OR, odds ratio; CI, confidence interval; AUC, area under the receiver operating characteristic curve; GAD, generalized anxiety disorder; PTSD, post-traumatic stress disorder; MDD, major depressive disorder; ADHD, attention-deficit/hyperactivity disorder; ODD, oppositional-defiant disorder; IED, intermittent explosive disorder.

a Pre-matriculation-onset disorders were defined as those with onsets at ages $0-17$ years.

${ }^{\mathrm{b}}$ Post-matriculation-onset disorders were defined as those with onsets at ages $18+$ years.

${ }^{\mathrm{c}}$ Respondents were limited to those in the part II sample who were 18-22 years old at the time of interview and had not graduated from college.

${ }^{\mathrm{d}}$ Prevalence of post-matriculation onsets was estimated in the subsample of respondents who did not have a pre-matriculation history of the disorder. As a result, the sample size for each calculation among students varies across disorders from a low of 1336 who had no pre-matriculation history of specific phobias to a high of 1563 who had no prematriculation history of drug dependence, with a median sample size of 1548 . The sample size for having no pre-matriculation history of any disorder is 1102 among students. Among attriters, the sample size for estimating prevalence of post-matriculation onsets for respondents who did not have a pre-matriculation history of the disorder varies from a low of 574 among attriters who had no pre-matriculation history of specific phobias to a high of 690 among attriters who had no pre-matriculation history of GAD, with a median sample size of 676. The sample size for having no pre-matriculation history of any disorder is 429 among attriters.

${ }^{\mathrm{e}}$ Based on a pooled logistic model in which a dummy variable for the pre-matriculation disorder in the row heading or, in the case of part VI, a count of number of prematriculation disorders, was used to predict whether the respondent was a student (coded 1) or an attriter (coded 0) within the sample of respondents who entered college, controlling age-sex and survey.

${ }^{\mathrm{f}}$ Based on a pooled logistic model in which a dummy variable for the post-matriculation disorder in the row heading among respondents who did not have that disorder prior to matriculation or, in the case of part VI, a count of number of pre-matriculation disorders in the total sample, was used to predict whether the respondent was a student (coded 1) or an attriter (coded 0) within the sample of respondents who entered college, controlling age-sex and survey.

${ }^{g}$ There were no post-matriculation onsets of these disorders by definition, as the CIDI required onsets before the age of 8 years (ADHD) or 18 years (conduct disorder, ODD).

${ }^{\mathrm{h}}$ Prevalence of post-matriculation onsets was estimated in the subsample of respondents who did not have a pre-matriculation history of the disorder. As a result, the sample size for each calculation varies across disorders from a low of 653 among male students and 683 among female students who had no pre-matriculation history of specific phobias to a high of 742 among male students and 821 among female students who had no pre-matriculation history of drug dependence. The sample size for having no pre-matriculation history of any disorder is 544 among male students and 558 among female students.

${ }^{1}$ Prevalence of post-matriculation onsets was estimated in the subsample of respondents who did not have a pre-matriculation history of the disorder. As a result, the sample size for each calculation varies across disorders from a low of 239 among male attriters and 335 among female attriters who had no pre-matriculation history of specific phobias to a high of 267 among male attriters and 425 among female attriters who had no pre-matriculation history of panic disorder. The sample size for having no pre-matriculation history of any disorder is 170 among male attriters and 259 among female attriters.

* Significant $(p<0.05$, two-sided test). 
Table 5. Pooled proportions of respondents with 12-month DSM-IVICIDI mental disorders who received minimally adequate treatment for these disorders in the 12 months before interview by country income level separately among respondents who were current students, college attriters and non-students in the same age range ${ }^{\mathrm{a}}$

\begin{tabular}{|c|c|c|c|c|c|c|}
\hline & \multicolumn{2}{|l|}{ Students } & \multicolumn{2}{|l|}{ Attriters } & \multicolumn{2}{|l|}{ Others } \\
\hline & $\%$ (S.E.) & $n$ & $\%$ (S.E.) & $n$ & $\%$ (S.E.) & $n$ \\
\hline Low/lower-middle & $6.7(3.9)$ & 79 & $2.2(2.2)$ & 28 & $6.1(2.8)$ & 206 \\
\hline Upper-middle & $11.4(5.1)$ & 60 & $9.4(4.9)$ & 30 & $8.5(2.2)$ & 237 \\
\hline High & $23.1(3.4)$ & 128 & $21.5(4.7)$ & 123 & $15.8(2.2)$ & 260 \\
\hline Total & $16.4(2.4)$ & 267 & $16.6(3.3)$ & 181 & $10.6(1.4)$ & 703 \\
\hline$\chi_{2}^{2}$ & $7.4^{*}$ & & $7.9^{*}$ & & $6.7^{*}$ & \\
\hline
\end{tabular}

DSM-IV, Diagnostic and Statistical Manual of Mental Disorders, 4th edition; CIDI, Composite International Diagnostic Interview; S.E., standard error.

${ }^{\text {a }}$ Respondents were limited to those in the part II sample who were 18-22 years old at the time of interview and had not graduated from college.

* Significant $(p<0.05$, two-sided test).

these (or other) joint predictors account for the associations of pre-matriculation mental disorders with college attrition, interventions to detect and treat these pre-existing disorders among entering freshmen would not reduce subsequent attrition. On the other hand, to the extent that the pre-matriculation disorders associated with college attrition represent common causal pathways for many of the more traditional predictors of attrition documented by educational researchers, interventions focused on college entrants with these pre-existing disorders might be of great value in reducing attrition.

There is no definitive way to adjudicate between these competing possibilities with the data presented here. A definitive evaluation would require experimental intervention where students with pre-matriculation disorders were detected and randomized either into best-practice treatments or usual care. Such an intervention would be most feasible in high-income countries, where access to out-patient mental health treatment in high, even though there is wide variation in the number of out-patient mental health workers per 100000 people in the populations of these countries (e. g. 23 in Portugal; 40-46 in the Netherlands, Poland and Spain; 125 in the USA; 158 in France) (World Health Organization, 2015). Given the much lower availability of out-patient mental health treatment providers in upper-middle- and lower-middle/low-income countries (e.g. 9.5/100000 in Mexico and 0.9/100000 in Nigeria), it seems unrealistic to think that resources would be available to provide out-patient treatment for college students in such countries.

In carrying out such an intervention, a question would arise whether the intervention should be limited to active disorders or also include relapse prevention for students with lifetime disorders that were not active at the time of matriculation. The results presented here are moot on this point because the WMH surveys did not collect data on whether disorders with prematriculation onsets were active or remitted at the time of college entry. However, it is noteworthy that 12-month prevalence of the pre-matriculation lifetime disorders associated with subsequent attrition was generally not more common among attriters than students. If pre-matriculation lifetime disorders were causal risk factors rather than risk markers for subsequent attrition, we would expect that 12-month presence of these same disorders would predict subsequent attrition, in which case our failure to find higher 12-month prevalence among attriters than students might be taken as evidence that these prematriculation disorders were risk markers rather than causal risk factors. It is important to recognize, though, that questions about disorder recency were not sufficiently specific in the WMH surveys to allow us to determine prevalence in the 12 months prior to attrition. As a result, our failure to find higher 12-month prevalence among attriters than students could be due to the consequences of attrition (e.g. being dismissed from college leading to a reduction in the heavy substance use that led to the dismissal). A similar kind of reciprocal influence might account for the fact that disorders with post-matriculation onsets were not more common among attriters than students.

Several limitations of our study are noteworthy. Some of these involve sampling. Perhaps the most obvious one is that the number of college students in the $\mathrm{WMH}$ surveys was too small to support separate 
analyses in each country. We carried out some crossnational analyses comparing results in high-, uppermiddle-, and lower-middle/low-income countries, but this level of disaggregation was less complete than we would have been preferred. The small sample sizes also resulted in wide CIs of estimates, with many of the ORs considered significant having CIs where one bound was very close to 1.0 and in a number of cases rounded to 1.0 with a single decimal of precision. Another sampling-related limitation is that not all WMH surveys included group housing in their sample frames. This means that these surveys under-represented students living in campus dormitories or fraternitysorority houses. Most WMH surveys also failed to distinguish between students living in off-campus housing with their families $v$. with room-mates. These sample limitations have the potential to be important in that some mental disorders (e.g. separation anxiety disorder, substance disorders) are likely to be higher among students living away from home and possibly more so in group housing than elsewhere.

Another set of limitations involves the assessment of mental disorders. Lifetime mental disorders and ages-of-onset were assessed retrospectively. Although the $\mathrm{WMH}$ assessment used procedures shown experimentally to improve accuracy of recall (Scott et al. 2014), there are likely to be downward biases in reports of lifetime prevalence and possibly also in dating age-of-onset of disorders due to recall errors. It is unclear whether these biases would be different for students, attriters and respondents who never entered college. In addition, diagnoses were based on fully structured lay-administered interviews rather than semi-structured clinical interviews, although the WMH clinical appraisal data are reassuring (Haro et al. 2006). In addition, we assessed only prevalence, not severity, of disorders. This omission could be important given that other research has shown that educational attainment is associated not only with prevalence but also severity of mental disorders (Ten Have et al. 2013).

A third set of limitations involves the imprecision in our information on timing of disorder onset, matriculation and attrition. This imprecision made it impossible for us to draw firm distinctions between mental disorders that occurred before $v$. after matriculation or, in the case of disorders with clear postmatriculation onsets, those that occurred before $v$. subsequent to attrition. Importantly, we dealt with this limitation by erring on the side of being conservative by considering only disorders with onsets prior to the age of 18 years as being prematriculation disorders. As a result, correction of this problem would only lead to an increase in the strength of our finding that the vast majority of the disorders of college students are pre-matriculation disorders.

Despite these limitations, our results are clear in showing that mental disorders are common among college students around the world, that the vast majority of these disorders have pre-matriculation onsets (which, if anything, were under-estimated by our lack of precision in the dating of matriculation), and that pre-matriculation mental disorders are associated both with failure to enter college and with attrition. We also found that only a small minority of college students with mental disorders receive even minimally adequate treatment and that the treatment rate is even lower in low-income countries. These results could have important human capital implications that would make it cost-effective from a societal perspective to invest in screening and increased treatment of college student mental disorders.

\section{Supplementary material}

The supplementary material for this article can be found at http://dx.doi.org/10.1017/S0033291716001665

\section{Acknowledgements}

The WHO WMH Survey Initiative is supported by the National Institute of Mental Health (NIMH; R01 MH070884), the John D. and Catherine T. MacArthur Foundation, the Pfizer Foundation, the US Public Health Service (R13-MH066849, R01-MH069864 and R01 DA016558), the Fogarty International Center (FIRCA R03-TW006481), the Pan American Health Organization (PAHO), Eli Lilly and Company, Ortho-McNeil Pharmaceutical, GlaxoSmithKline and Bristol-Myers Squibb. We thank the staff of the WMH Data Collection and Data Analysis Coordination Centres for assistance with instrumentation, fieldwork and consultation on data analysis. None of the funders had any role in the design, analysis, interpretation of results, or preparation of this paper. This report was prepared under the auspices of the WHO International Classification of Diseases-11, chapter 5 (Mental and Behavioural Disorders) epidemiology working group, which is co-chaired by Somnath Chatterji and Ronald C. Kessler. The views and opinions expressed in this report are those of the authors and should not be construed to represent the views of the sponsoring organizations, agencies or governments. The 2007 Australian National Survey of Mental Health and Wellbeing is funded by the Australian Government Department of Health and Ageing. The São Paulo Megacity Mental Health Survey is supported by the State of São Paulo Research Foundation (FAPESP) 
Thematic Project Grant 03/00204-3. The Bulgarian Epidemiological Study of common mental disorders (EPIBUL) is supported by the Ministry of Health and the National Center for Public Health Protection. The Chinese WMH Survey Initiative is supported by the Pfizer Foundation. The Shenzhen Mental Health Survey is supported by the Shenzhen Bureau of Health and the Shenzhen Bureau of Science, Technology, and Information. The Colombian National Study of Mental Health (NSMH) is supported by the Ministry of Social Protection. The Mental Health Study Medellín - Colombia was carried out and supported jointly by the Center for Excellence on Research in Mental Health (CES University) and the Secretary of Health of Medellín. The ESEMeD project is funded by the European Commission (contracts QLG5-1999-01042; SANCO 2004123 and EAHC 20081308), the Piedmont Region (Italy), Fondo de Investigación Sanitaria, Instituto de Salud Carlos III, Spain (FIS 00/0028), Ministerio de Ciencia y Tecnología, Spain (SAF 2000-158-CE), Departament de Salut, Generalitat de Catalunya, Spain, Instituto de Salud Carlos III (CIBER CB06/02/0046, RETICS RD06/0011 REM-TAP), and other local agencies and by an unrestricted educational grant from GlaxoSmithKline. Implementation of the Iraq Mental Health Survey (IMHS) and data entry were carried out by the staff of the Iraqi Ministries of Health and Planning with direct support from the Iraqi IMHS team with funding from both the Japanese and European Funds through the United Nations Development Group Iraq Trust Fund. The Lebanese National Mental Health Survey (L.E.B.A.N.O.N.) is supported by the Lebanese Ministry of Public Health, the WHO (Lebanon), National Institutes of Health/ Fogarty International Center (R03 TW006481-01), the Sheikh Hamdan Bin Rashid Al Maktoum Award for Medical Sciences, anonymous private donations to IDRAAC, Lebanon, and unrestricted grants from AstraZeneca, Eli Lilly, GlaxoSmithKline, Hikma Pharmaceuticals, Janssen Cilag, Lundbeck, Novartis and Servier. The Mexican National Comorbidity Survey is supported by The National Institute of Psychiatry Ramon de la Fuente (INPRFMDIES 4280) and by the National Council on Science and Technology (CONACyT-G30544-H), with supplemental support from the PAHO. C.B. has received funding from the (Mexican) National Council of Science and Technology (grant CB-2010-01-155221). Te Rau Hinengaro: The New Zealand Mental Health Survey is supported by the New Zealand Ministry of Health, Alcohol Advisory Council and the Health Research Council. The Nigerian Survey of Mental Health and Wellbeing is supported by the WHO (Geneva), the WHO (Nigeria) and the Federal Ministry of Health,
Abuja, Nigeria. The Northern Ireland Study of Mental Health was funded by the Health \& Social Care Research \& Development Division of the Public Health Agency. The Peruvian WMH Study was funded by the National Institute of Health of the Ministry of Health of Peru. The Polish project Epidemiology of Mental Health and Access to Care EZOP Poland - was carried out by the Institute of Psychiatry and Neurology in Warsaw in consortium with the Department of Psychiatry - Medical University in Wroclaw and the National Institute of Public Health-National Institute of Hygiene in Warsaw and in partnership with Psykiatrist Institut Vinderen - Universitet, Oslo. The project was funded by the Norwegian Financial Mechanism and the European Economic Area Mechanism as well as the Polish Ministry of Health. No support from the pharmaceutical industry was received or from other commercial sources. The Portuguese Mental Health Study was carried out by the Department of Mental Health, Faculty of Medical Sciences, NOVA University of Lisbon, with collaboration of the Portuguese Catholic University, and was funded by the Champalimaud Foundation, Gulbenkian Foundation, Foundation for Science and Technology and the Ministry of Health. The Romania WMH study projects 'Policies in Mental Health Area' and 'National Study regarding Mental Health and Services Use' were carried out by the National School of Public Health \& Health Services Management (former National Institute for Research \& Development in Health), with technical support of Metro Media Transylvania, the National Institute of Statistics-National Centre for Training in Statistics, SC Cheyenne Services SRL, Statistics Netherlands and were funded by the Ministry of Public Health (former Ministry of Health) with supplemental support of Eli Lilly Romania SRL. The US National Comorbidity Survey Replication is supported by the NIMH (U01-MH60220) with supplemental support from the National Institute of Drug Abuse, the Substance Abuse and Mental Health Services Administration, the Robert Wood Johnson Foundation (grant 044708) and the John W. Alden Trust. A complete list of all within-country and cross-national WMH publications can be found at http://www.hcp.med.harvard.edu/ $\mathrm{wmh} /$. The views and opinions expressed in this article are those of the authors and should not be construed to represent the views of any of the sponsoring organizations, agencies or governments.

The WHO WMH Survey Collaborators are Tomasz Adamowski, Ph.D., M.D., Sergio Aguilar-Gaxiola, M.D., Ph.D., Ali Al-Hamzawi, M.D., Mohammad Al-Kaisy, M.D., Abdullah Al Subaie, F.R.C.P., Jordi Alonso, M.D., Ph.D., Yasmin Altwaijri, M.S., Ph.D., 
Laura Helena Andrade, M.D., Ph.D., Lukoye Atwoli, M.D., Randy Auerbach, Ph.D., William Axinn, Ph.D., Corina Benjet, Ph.D., Guilherme Borges, Sc.D., Robert Bossarte, Ph.D., Evelyn Bromet, Ph.D., Ronny Bruffaerts, Ph.D., Brendan Bunting, Ph.D., Ernesto Caffo, M.D., Jose Miguel Caladas de Almeida, M.D., Ph.D., Graca Cardoso, M.D., Ph.D., Stephanie Chardoul, Somnath Chatterji, M.D., Alexandre Chiavegatto Filho, Ph.D., Pim Cuijpers, Ph.D., Louisa Degenhardt, Ph.D., Giovanni de Girolamo, M.D., Ron deGraaf, M.S., Ph.D., Peter deJonge, Ph.D., Koen Demyttenaere, M.D., Ph.D., David Ebert, Ph.D., Sara Evans-Lacko, Ph.D., John Fayyad, M.D., Marina Piazza Ferrand, DSc, M.P.H., Fabian Fiestas, M.D., Ph.D., Silvia Florescu, M.D., Ph.D., Barbara Forresi, Ph.D., Sandro Galea, Dr.P.H., M.D., M.P.H., Laura Germine, Ph.D., Stephen Gilman, Sc.D., Dirgha Ghimire, Ph.D., Oye Gureje, M.D., Ph.D., Josep Maria Haro, M.D., M.P.H., Ph.D., Yanling He, M.D., Hristo Hinkov, M.D., Chi-yi Hu, Ph.D., M.D., Yueqin Huang, M.D., M.P.H., Ph.D., Aimee Nasser Karam, Ph.D., Elie G. Karam, M.D., Norito Kawakami, M.D., Ph.D., Andrzej Kiejna, Ph.D., M.D., Karestan Koenen, Ph.D., Viviane Kovess-Masfety, M.S., M.D., Ph.D., Luise Lago, Ph.D., Carmen Lara, M.D., Ph.D., Sing Lee, Ph.D., Jean-Pierre Lepine, M.D., Itzhak Levav, M.D., Daphna Levinson, Ph.D., Zhaorui Liu, M.D., M.P.H., Silvia Martins, M.D., Ph.D., Herbert Matschinger, Ph.D., John McGrath, Ph.D., Katie McLaughlin, Ph.D., Maria Elena Medina-Mora, Ph.D., Zeina Mneimneh, Ph.D., M.P.H., Jacek Moskalewicz, Dr.P.H., Samuel Murphy, Dr.P.H., Fernando Navarro-Mateu, M.D., Ph.D., Matt Nock, Ph.D., Siobhan O'Neill, Ph.D., Mark Oakley-Browne, M.B., Ch.B., Ph.D., FRANZCP, J. Hans Ormel, Ph.D., Beth-Ellen Pennell, M.A., Stephanie Pinder-Amaker, Ph.D., Patryk Piotrowski, M.D., Ph.D., Jose Posada-Villa, M.D., Ayelet Ruscio, Ph.D., Kate Scott, Ph.D., Vicki Shahly, Ph.D., Derrick Silove, Ph.D., Tim Slade, Ph.D., Jordan Smoller, Sc.D., M.D., Dan J Stein, M.B.A., M.Sc., Ph.D., Amy Street, Ph.D., Hisateru Tachimori, Ph.D., Nezar Taib, M.S., Margreet ten Have, Ph.D., Graham Thornicroft, M.D., Yolanda Torres de Galvis, M.P.H., Maria Carmen Viana, M.D., Ph.D., Gemma Vilagut, M.S., Elisabeth Wells, Ph.D., David R. Williams, M.P.H., Ph.D., Michelle Williams, Sc.D., Bogdan Wojtyniak, Sc.D., Alan Zaslavsky, Ph.D. The WHO WMH Survey collaborators are Tomasz Adamowski, Ph.D., M.D., Sergio Aguilar-Gaxiola, M.D., Ph.D., Ali Al-Hamzawi, M.D., Mohammad Al-Kaisy, M.D., Abdullah Al Subaie, M. B.B.S., F.R.C.P., Jordi Alonso, M.D., Ph.D., Yasmin Altwaijri, M.S., Ph.D., Laura Helena Andrade, M.D., Ph.D., Lukoye Atwoli, M.D., Randy P. Auerbach, Ph. D., William G. Axinn, Ph.D., Corina Benjet, Ph.D.,
Guilherme Borges, Sc.D., Robert M. Bossarte, Ph.D., Evelyn J. Bromet, Ph.D., Ronny Bruffaerts, Ph.D., Brendan Bunting, Ph.D., Ernesto Caffo, M.D., Jose Miguel Caldas de Almeida, M.D., Ph.D., Graca Cardoso, M.D., Ph.D., Alfredo H. Cia, M.D., Stephanie Chardoul, Somnath Chatterji, M.D., Alexandre Chiavegatto Filho, Ph.D., Pim Cuijpers, Ph.D., Louisa Degenhardt, Ph.D., Giovanni de Girolamo, M.D., Ron de Graaf, M.S., Ph.D., Peter de Jonge, Ph.D., Koen Demyttenaere, M.D., Ph.D., David D. Ebert, Ph.D., Sara Evans-Lacko, Ph.D., John Fayyad, M.D., Fabian Fiestas, M.D., Ph.D., Silvia Florescu, M.D., Ph.D., Barbara Forresi, Ph.D., Sandro Galea, Dr.P.H., M.D., M.P.H., Laura Germine, Ph.D., Stephen E. Gilman, Sc.D., Dirgha J. Ghimire, Ph.D., Meyer D. Glantz, Ph.D., Oye Gureje, Ph.D., DSc, F.R. C.Psych., Josep Maria Haro, M.D., M.P.H., Ph.D., Yanling He, M.D., Hristo Hinkov, M.D., Chi-yi Hu, Ph.D., M.D., Yueqin Huang, M.D., M.P.H., Ph.D., Aimee Nasser Karam, Ph.D., Elie G. Karam, M.D., Norito Kawakami, M.D., D.M.Sc., Ronald C. Kessler, Ph.D., Andrzej Kiejna, M.D., Ph.D., Karestan C. Koenen, Ph.D., Viviane Kovess-Masfety, M.Sc., M.D., Ph.D., Luise Lago, Ph.D., Carmen Lara, M.D., Ph.D., Sing Lee, Ph.D., Jean-Pierre Lepine, M.D., Itzhak Levav, M.D., Daphna Levinson, Ph.D., Zhaorui Liu, M.D., M.P.H., Silvia S. Martins, M.D., Ph.D., Herbert Matschinger, Ph.D., John J. McGrath, Ph.D., Katie A. McLaughlin, Ph.D., Maria Elena Medina-Mora, Ph.D., Zeina Mneimneh, Ph.D., M.P. H., Jacek Moskalewicz, Dr.P.H., Samuel D. Murphy, Dr.P.H., Fernando Navarro-Mateu, M.D., Ph.D., Matthew K. Nock, Ph.D., Siobhan O'Neill, Ph.D., Mark Oakley-Browne, M.B., Ch.B., Ph.D., J. Hans Ormel, Ph.D., Beth-Ellen Pennell, M.A., Marina Piazza, M.P.H., Sc.D., Stephanie Pinder-Amaker, Ph. D., Patryk Piotrowski, M.D., Ph.D., Jose Posada-Villa, M.D., Ayelet M. Ruscio, Ph.D., Kate M. Scott, Ph.D., Vicki Shahly, Ph.D., Derrick Silove, Ph.D., Tim Slade, Ph.D., Jordan W. Smoller, Sc.D., M.D., Juan Carlos Stagnaro, M.D., Ph.D., Dan J. Stein, M.B.A., M.Sc., Ph.D., Amy E. Street, Ph.D., Hisateru Tachimori, Ph.D., Nezar Taib, M.S., Margreet ten Have, Ph.D., Graham Thornicroft, Ph. D., Yolanda Torres, M.P.H., Maria Carmen Viana, M.D., Ph.D., Gemma Vilagut, M.S., Elisabeth Wells, Ph.D., David R. Williams, M.P.H., Ph.D., Michelle A. Williams, Sc.D., Bogdan Wojtyniak, Sc.D. and Alan M. Zaslavsky, Ph.D.

R.P.A., R.B., P.C., D.D.E. and R.C.K. conceived of the paper and designed the analysis plan. R.C.K. and N.A. S. supervised the statistical analysis, which was carried out by I.H and H.L. All co-authors participated in discussions to interpret the results. R.P.A. and R.C.K. wrote the first draft, while all other co-authors 
participated in revisions and approved the final version of the manuscript for submission.

\section{Declaration of Interest}

In the past 3 years, R.C.K. has served as a consultant for or received research support from Johnson \& Johnson Wellness and Prevention, the Lake Nona Life Project and Shire Pharmaceuticals. R.C.K. is a coowner of DataStat, Inc., a market research company that carries out healthcare research. The other authors report no biomedical financial interests or potential conflicts of interest. K.D. has served on advisory boards and speaker bureaus with Astra Zeneca, Eli Lilly, Lundbeck, Johnson \& Johnson, Naurex and Servier. K.D. has received grants from Eli Lilly, Fonds Ga Voor Geluk and Fonds voor Wetenschappelijk Onderzoek Vlaandereden.

\section{References}

Blanco C, Okuda M, Wright C, Hasin DS, Grant BF, Liu SM, Olfson M (2008). Mental health of college students and their non-college-attending peers: results from the National Epidemiologic Study on Alcohol and Related Conditions. Archives of General Psychiatry 65, 1429-1437.

Cho SB, Llaneza DC, Adkins AE, Cooke M, Kendler KS, Clark SL, Dick DM (2015). Patterns of substance use across the first year of college and associated risk factors. Frontiers in Psychiatry 6, 152.

Costello EJ, Egger H, Angold A (2005). 10-Year research update review: the epidemiology of child and adolescent psychiatric disorders: I. Methods and public health burden. Journal of the American Academy of Child and Adolescent Psychiatry 44, 972-986.

Costello EJ, Pine DS, Hammen C, March JS, Plotsky PM, Weissman MM, Biederman J, Goldsmith HH, Kaufman J, Lewinsohn PM, Hellander M, Hoagwood K, Koretz DS, Nelson CA, Leckman JF (2002). Development and natural history of mood disorders. Biological Psychiatry 52, 529-542.

Eisenberg D, Golberstein E, Hunt J (2009). Mental health and academic success in college. B.E. Journal of Economic Analysis and Policy 9, 40.

Eisenberg D, Gollust SE, Golberstein E, Hefner JL (2007). Prevalence and correlates of depression, anxiety, and suicidality among university students. American Journal of Orthopsychiatry 77, 534-542.

Fazel M, Hoagwood K, Stephan S, Ford T (2014a). Mental health interventions in schools 1 : mental health interventions in schools in high-income countries. Lancet Psychiatry 1, 377-387.

Fazel M, Patel V, Thomas S, Tol W (2014b). Mental health interventions in schools in low-income and middle-income countries. Lancet Psychiatry 1, 388-398.

Fergusson DM, Horwood LJ (1998). Early conduct problems and later life opportunities. Journal of Child Psychology and Psychiatry, and Allied Disciplines 39, 1097-1108.
Fergusson DM, Woodward LJ (2002). Mental health, educational, and social role outcomes of adolescents with depression. Archives of General Psychiatry 59, 225-231.

First M, Spitzer R, Gibbon M, Williams B (1994). Structured Clinical Interview for Axis I DSM-IV Disorders. New York State Psychiatric Institute: Biometrics Research Department: New York.

Fletcher JM (2008). Adolescent depression: diagnosis, treatment, and educational attainment. Health Economics 17, 1215-1235.

Harkness J, Pennell B, Villar A, Gebler N, Aguilar-Gaxiola S, Bilgen I (2008). Translation procedures and translation assessment in the World Mental Health Survey Initiative. In The WHO World Mental Health Surveys: Global Perspectives on the Epidemiology of Mental Disorders (ed. R. C. Kessler), pp. 91-113. Cambridge University Press: New York.

Haro JM, Arbabzadeh-Bouchez S, Brugha TS, de Girolamo G, Guyer ME, Jin R, Lepine JP, Mazzi F, Reneses B, Vilagut G, Sampson NA, Kessler RC (2006). Concordance of the Composite International Diagnostic Interview version 3.0 (CIDI 3.0) with standardized clinical assessments in the WHO World Mental Health surveys. International Journal of Methods in Psychiatric Research 15, 167-180.

Hartley MT (2010). Increasing resilience: strategies for reducing dropout rates for college students with psychiatric disabilities. American Journal of Psychiatric Rehabilitation 13, 295-315.

Heeringa S, Wells E, Hubbard F, Mneimneh Z, Chiu W, Sampson N, Berglund P (2008). Sample designs and sampling procedures. In The WHO World Mental Health Surveys: Global Perspectives on the Epidemiology of Mental Disorders (ed. R. C. Kessler), pp. 14-32. Cambridge University Press: New York.

Hunt J, Eisenberg D, Kilbourne AM (2010). Consequences of receipt of a psychiatric diagnosis for completion of college. Psychiatric Services 61, 399-404.

Ishitani T, DesJardins S (2002). A longitudinal investigation of dropout from college in the United States. Journal of College Student Retention: Research, Theory and Practice 4, 173-201.

Johnson JG, Cohen P, Dohrenwend BP, Link BG, Brook JS (1999). A longitudinal investigation of social causation and social selection processes involved in the association between socioeconomic status and psychiatric disorders. Journal of Abnormal Psychology 108, 490-499.

Kendler KS, Myers J, Dick D (2015). The stability and predictors of peer group deviance in university students. Social Psychiatry and Psychiatric Epidemiology 50, 1463-1470.

Kessler R, Üstun T (2011). The WHO World Mental Health Surveys: Global Perspectives on the Epidemiology of Mental Disorders. Cambridge University Press: Cambridge.

Kessler RC, Akiskal HS, Angst J, Guyer M, Hirschfeld RM, Merikangas KR, Stang PE (2006). Validity of the assessment of bipolar spectrum disorders in the WHO CIDI 3.0. Journal of Affective Disorders 96, 259-269.

Kessler RC, Foster CL, Saunders WB, Stang PE (1995). Social consequences of psychiatric disorders, I: educational attainment. American Journal of Psychiatry 152, 1026-1032. 
Kessler RC, Ustun TB (2004). The World Mental Health (WMH) Survey Initiative version of the World Health Organization (WHO) Composite International Diagnostic Interview (CIDI). International Journal of Methods in Psychiatric Research 13, 93-121.

Kilpatrick DG, Acierno R, Saunders B, Resnick HS, Best CL, Schnurr PP (2000). Risk factors for adolescent substance abuse and dependence: data from a national sample. Journal of Consulting and Clinical Psychology 68, 19-30.

Knäuper B, Cannell CF, Schwarz N, Bruce ML, Kessler RC (1999). Improving accuracy of major depression age-of-onset reports in the US National Comorbidity Survey. International Journal of Methods in Psychiatric Research 8, 39-48.

Kraemer HC, Kazdin AE, Offord DR, Kessler RC, Jensen PS, Kupfer DJ (1997). Coming to terms with the terms of risk. Archives of General Psychiatry 54, 337-343.

Lee S, Tsang A, Breslau J, Aguilar-Gaxiola $S$, Angermeyer M, Borges G, Bromet E, Bruffaerts R, de Girolamo G, Fayyad J, Gureje O, Haro JM, Kawakami N, Levinson D, Oakley Browne MA, Ormel J, Posada-Villa J, Williams DR, Kessler RC (2009). Mental disorders and termination of education in high-income and low- and middle-income countries: epidemiological study. British Journal of Psychiatry: the Journal of Mental Science 194, 411-417.

Lewinsohn PM, Rohde P, Seeley JR (1998). Major depressive disorder in older adolescents: prevalence, risk factors, and clinical implications. Clinical Psychology Review 18, 765-794.

Merikangas KR, Nakamura EF, Kessler RC (2009). Epidemiology of mental disorders in children and adolescents. Dialogues in Clinical Neuroscience 11, 7-20.

Miech RA, Caspi A, Moffitt TE, Wright BRE, Silva PA (1999). Low socioeconomic status and mental disorders: a longitudinal study of selection and causation during young adulthood. American Journal of Sociology 104, 1096-1131.

Mojtabai R, Stuart EA, Hwang I, Susukida R, Eaton WW, Sampson N, Kessler RC (2015). Long-term effects of mental disorders on employment in the national comorbidity survey ten-year follow-up. Social Psychiatry and Psychiatric Epidemiology 50, 1657-1668.

SAS Institute Inc. (2010). SAS/STATR Software. SAS Institute Inc.: Cary, NC.

Scott KM, Al-Hamzawi AO, Andrade LH, Borges G, Caldas-de-Almeida JM, Fiestas F, Gureje O, Hu C, Karam EG, Kawakami N, Lee S, Levinson D, Lim CC,

Navarro-Mateu F, Okoliyski M, Posada-Villa J, Torres $Y$, Williams DR, Zakhozha V, Kessler RC (2014).

Associations between subjective social status and DSM-IV mental disorders: results from the World Mental Health Surveys. JAMA Psychiatry 71, 1400-1408.

Stratton SL, O'Toole MD, Wetzel NJ (2006). Are the factors affecting dropout behavior related to initial enrollment intensity for college undergraduates? Research in Higher Education 48, 453-485.

Ten Have M, Nuyen J, Beekman A, de Graaf R (2013). Common mental disorder severity and its association with treatment contact and treatment intensity for mental health problems. Psychological Medicine 43, 2203-2213.

The World Bank (2012). Data: countries and economies (http://data.worldbank.org/country).

Wang PS, Aguilar-Gaxiola S, Alonso J, Angermeyer MC, Borges G, Bromet EJ, Bruffaerts R, de Girolamo G, de Graaf R, Gureje O, Haro JM, Karam EG, Kessler RC, Kovess V, Lane MC, Lee S, Levinson D, Ono $Y$, Petukhova M, Posada-Villa J, Seedat S, Wells JE (2007). Use of mental health services for anxiety, mood, and substance disorders in 17 countries in the WHO World Mental Health surveys. Lancet 370, 841-850.

Wolter K (1985). Introduction to Variance Estimation. Springer-Verlag: New York.

Woodward LJ, Fergusson DM (2001). Life course outcomes of young people with anxiety disorders in adolescence. Journal of the American Academy of Child and Adolescent Psychiatry 40, 1086-1093.

World Health Organization (2015). Mental Health Atlas 2014. World Health Organization: Geneva. 